\title{
Dosage Estimation in Mammography for Quality Assurance and Quality Control Using Adult Mammograms
}

\author{
Oladotun Ayotunde Ojo ${ }^{1,}$, , Musibau Adekunle Ibrahim², Peter Adefisoye Oluwafisoye ${ }^{1}$, \\ Charles Okechukwu Chime ${ }^{3}$ \\ ${ }^{1}$ Department of Physics, Osun State University, Osogbo, Nigeria \\ ${ }^{2}$ Department of Information and Communication Technology, Osun State University, Osogbo, Nigeria \\ ${ }^{3}$ Department of Radiology, University of Benin Teaching Hospital, Benin City, Nigeria
}

Email address:

oladotun.ojo@uniosun.edu.ng (O. A. Ojo)

${ }^{*}$ Corresponding author

\section{To cite this article:}

Oladotun Ayotunde Ojo, Musibau Adekunle Ibrahim, Peter Adefisoye Oluwafisoye, Charles Okechukwu Chime. Dosage Estimation in Mammography for Quality Assurance and Quality Control Using Adult Mammograms. Radiation Science and Technology.

Vol. 4, No. 2, 2018, pp. 6-11. doi: 10.11648/j.rst.20180402.11

Received: July 17, 2018; Accepted: August 22, 2018; Published: September 17, 2018

\begin{abstract}
Any form of X-ray exposure should be carefully monitored and controlled, so that the patient is only exposed to safe amounts. Even though X-ray users are extremely careful when exposing their patients to diagnostic tests, where radiation is involved, it is important to bear in mind how low the risks really are, especially when compared to other forms of radiation exposure. As with any kind of medical procedure, X-rays are safe when they are used properly. When clinically indicated, properly conducted imaging with the smallest risk should be performed. In this study, the optical densities, OD, of adult mammograms, undergoing X-ray exposures was measured from the radiology department of the University of Benin Teaching Hospital (UBTH), Benin City, Edo State, Nigeria, with the aid of a densitometer, model MA 5336. The measured optical densities were used to estimate the X-ray radiation dose to patients, undergoing mammography, for the purpose of Quality Assurance (QA) and Quality Control (QC), in diagnostic and screening. A total of fifty (50) adult mammograms were collected for use in the radiology department of the hospital. The optical densities were measured five times at different spots across the image of each of the mammograms and the mean were obtained, in other to estimate the absorbed dose. The results obtained showed that the mean dose was $0.48 \mathrm{mGy}$, minimum dose $0.04 \mathrm{mGy}$, maximum dose $1.07 \mathrm{mGy}$, range of dose $1.03 \mathrm{mGy}$, SD 0.24 , kurtosis $2.77,1^{\text {st }} \mathrm{Q} 0.34$ and $3^{\text {rd }} \mathrm{Q} 0.66$. These results were in agreement with those of the International Atomic Energy Agency (IAEA), guidance levels in X-ray guided medical interventional procedures.
\end{abstract}

Keywords: Exposure, Radiation, Absorbed Dose, Mammograms, Optical Densities

\section{Introduction}

Mammography is specialized medical imaging that uses a low-dose X-ray system to see inside the breasts. A mammography exam, called a mammogram, aids in the early detection and diagnosis of breast diseases in women. An Xray (radiograph) is a noninvasive medical test that helps physicians diagnose and treat medical conditions. Imaging with X-rays involves exposing a part of the body to a small dose of ionizing radiation to produce pictures of the inside of the body. X-rays are the oldest and most frequently used form of medical imaging. Three recent advances in mammography include digital mammography, computeraided detection and breast tomosynthesis. Although the radiation dose for some breast tomosynthesis systems is slightly higher than the dosage used in standard mammography, it remains within the Food and Drug Administration (FDA) approved safe levels for radiation from mammograms. Some systems have doses very similar to conventional mammography. Medical exposure of man to ionizing radiation arises from practices such as diagnostic, therapeutic and nuclear medicine procedures. Consequently, the patients, medical radiation specialists and the general population receive significant exposure to ionizing radiation. 
Medical exposure to radiation, from artificial or man-made radiation sources, contributes the largest component of radiation dose to general population [1]. It has also been estimated that diagnostic radiology and nuclear medicine procedures contribute $88 \%$ of dose to the collective effective dose from man- made sources of radiation in the United State of America [2]. Patient's doses from medical X-ray examination in Nigeria as reported by [3] have shown large inter - and intra-hospital variations for similar radiological examinations. Recent in vitro experiments support the hypothesis that the radiation environment of space could also contribute to the long-term physiological changes astronauts experience after missions [4, 5]. Exposure from different sources has various total doses, exposure rates, linear energy transfer, and spectral features, which make certain aspects more harmful or more beneficial than others [6]. Welldefined characteristic X-rays produced by a novel X-ray fluorescence irradiation device were utilized to aid the physical characterization of the radiation, as standard X-ray tube sources produce a mix of Bremsstrahlung and characteristic emissions [7]. It is well accepted that one of the major problems in radiation research is how to extrapolate the plethora of published data on normal tissue damage and cancer risk assessment from high dose (HD) ionizing radiation (IR) (HDIR) exposures to low dose ionizing radiation (LDIR) range (generally, less than 0.1 Gy) [8]. The issue of cancer risks from imaging radiation has been a dominant theme in radiology for the past 15 years. It is now appropriate to review the current situation and the options for moving into the future [9]. Thus, presented the need to have a national standard for medical diagnostic and screening dose level for individual mammography in Nigeria for Quality Assurance (QA) and Quality Control (QC).

\section{Materials and Method}

Samples of adult X-ray mammograms were collected from UBTH, South - South zone of Nigeria. The following abbreviations were adopted for the purpose of this study:

Absorbed X-ray dose: X

Net optical density: NOD

Mean optical density: MOD

Optical density: OD or D

Measured optical densities: $\mathrm{OD}_{1}, \mathrm{OD}_{2}, \mathrm{OD}_{3}, \mathrm{OD}_{4}$ and $\mathrm{OD}_{5}$

Standard deviation: SD

Minimum absorbed dose: Min

Maximum absorbed dose: Max

First Quartile: $1^{\text {st }} \mathrm{Q}$

Third Quartile: $3^{\text {rd }} \mathrm{Q}$

Film serial number: FILM S/N

Table 1. Features of the densitometer (Gammex, 2016) [10].

\begin{tabular}{ll}
\hline Model & MA 5336 (made in USA by GAMMEX) \\
\hline Range & 0 to 4.0 optical density \\
Accuracy & \pm 0.02 density \\
Reproducibility & \pm 0.01 density \\
Warm up time & None \\
Measuring area & $2 \mathrm{~mm}$ diameter and $1 \mathrm{~mm}$ diameter \\
Power supply & Four rechargeable AA NiCad batteries, $4.8 \mathrm{~V}$ total rated at $600 \mathrm{mAh}$ (included) \\
Battery charger & SE $30-45(115 \mathrm{VAC})$ or SE $-30(230 \mathrm{VAC}) 50$ to $60 \mathrm{~Hz}$ \\
Charge time & Approximately 14 hours \\
Size & $5.08 \times 7.46 \mathrm{X} 17.8 \mathrm{~cm}(2 \mathrm{X} 2.9 \times 7 \mathrm{in})$ \\
Weight & $0.7 \mathrm{Kg}(1.5 \mathrm{lbs}$ ) \\
\hline
\end{tabular}

A film densitometer, model MA 5336 for the measurement of optical density was used. The light source/detector assembly is driven in finite incremental steps and a resolution over the entire scanning area to ensure precise positioning with a high degree of repeatability [11]. The film densitometer is a simple to use peripheral device for the measurement of the blackening density film exposed to ionizing radiation. Since X-ray image on the film is a black and white image with various blackening densities, the densitometer accepts standard X-ray films [11].

The optical densities of each mammograms, was measured repeatedly five times at different spots on each image of the film as optical densities $\mathrm{OD}_{1}, \mathrm{OD}_{2}, \mathrm{OD}_{3}, \mathrm{OD}_{4}$ and $\mathrm{OD}_{5}$. The average of the five optical densities was then taken to obtain the MOD. The optical densities were converted to the absorbed X-ray radiation doses $\mathrm{X}$, in milli gray (mGy), which is the amount of X-ray radiation dose that each patient was exposed to. The mean absorbed dose, range of absorbed dose, standard deviation, kurtosis, first and third quartiles were also calculated for the samples.
The blackening of the film after X-ray radiation exposure is expressed in terms of its optical density as [12]:

$$
D=\log _{10}\left(\frac{I_{O}}{I}\right)
$$

Where $I o$ and $I$ is the light intensities before and after passing the exposed film material. Optical density is a numerical value indicating the degree of blackening on an Xray radiographic film, and it is a dimensionless quantity. The correlation between the optical density $\mathrm{D}$ and the maximum number of sensitized grains results in a relation between the optical density $\mathrm{D}$ and the absorbed dose $\mathrm{X}$. Thus:

$$
D=D_{\max }\left[1-e^{-k X}\right]
$$

Where,

$D_{\max }=4$ (this is the maximum measurable OD obtainable with the densitometer) [12]

$\mathrm{k}=0.68$ [12]

Therefore, Equation (2) for the optical density that was measured becomes: 


$$
D=4\left[1-e^{-0.68 X}\right]
$$

Solving Equation (3) for the absorbed X-ray radiation dose $\mathrm{X}$, gives:

$$
X=\left(-\frac{1}{0.68}\right) \log _{e}\left(1-\frac{D_{M O D}}{4}\right)
$$

Equation (4) was used to convert the measured optical densities of each mammogram to absorbed X-ray radiation dose, in milli gray (mGy).

\section{Results and Discussion}

The results obtained in this work are presented in Tables 2 , 3 and 4 , for the mammogram examinations. Table 2 presents the measured film OD, NOD and the MOD.

\begin{tabular}{|c|c|c|c|c|c|c|c|}
\hline FILM S/N & $\mathbf{O D}_{1}$ & $\mathrm{OD}_{2}$ & $\mathrm{OD}_{3}$ & $\mathrm{OD}_{4}$ & $\mathrm{OD}_{5}$ & NOD & MOD \\
\hline 1 & 0.59 & 0.87 & 0.46 & 0.36 & 0.02 & 2.30 & 0.46 \\
\hline 2 & 0.79 & 0.94 & 1.90 & 1.92 & 1.30 & 6.85 & 1.37 \\
\hline 3 & 1.03 & 2.24 & 2.27 & 2.08 & 2.16 & 9.78 & 1.96 \\
\hline 4 & 1.92 & 2.21 & 2.04 & 2.01 & 2.18 & 10.36 & 2.07 \\
\hline 5 & 2.49 & 0.18 & 0.06 & 1.83 & 1.64 & 6.20 & 1.24 \\
\hline 6 & 0.17 & 0.08 & 0.07 & 0.04 & 0.22 & 0.58 & 0.12 \\
\hline 7 & 0.15 & 0.18 & 0.12 & 0.20 & 0.92 & 1.57 & 0.31 \\
\hline 8 & 0.04 & 0.91 & 0.01 & 0.06 & 0.01 & 1.03 & 0.21 \\
\hline 9 & 1.80 & 1.55 & 0.29 & 0.19 & 1.74 & 5.57 & 1.11 \\
\hline 10 & 1.03 & 3.22 & 1.43 & 1.40 & 1.12 & 8.20 & 1.64 \\
\hline 11 & 2.15 & 1.62 & 0.80 & 0.50 & 1.58 & 6.65 & 1.33 \\
\hline 12 & 0.23 & 0.74 & 0.17 & 0.29 & 0.31 & 1.74 & 0.35 \\
\hline 13 & 0.24 & 0.11 & 0.09 & 0.10 & 0.09 & 0.63 & 0.13 \\
\hline 14 & 0.18 & 0.10 & 1.26 & 0.60 & 1.18 & 3.32 & 0.66 \\
\hline 15 & 0.16 & 0.12 & 1.86 & 1.62 & 1.40 & 5.16 & 1.03 \\
\hline 16 & 1.66 & 1.74 & 0.01 & 0.09 & 2.09 & 5.59 & 1.12 \\
\hline 17 & 2.21 & 1.92 & 1.39 & 0.07 & 1.85 & 7.44 & 1.49 \\
\hline 18 & 1.92 & 1.93 & 1.23 & 1.76 & 0.12 & 6.96 & 1.39 \\
\hline 19 & 2.16 & 0.08 & 1.10 & 2.31 & 1.64 & 7.29 & 1.46 \\
\hline 20 & 1.41 & 1.74 & 1.39 & 3.64 & 0.69 & 8.87 & 1.77 \\
\hline 21 & 2.15 & 0.11 & 0.01 & 0.18 & 2.16 & 4.61 & 0.92 \\
\hline 22 & 2.29 & 0.13 & 0.23 & 0.15 & 1.18 & 3.98 & 0.80 \\
\hline 23 & 0.26 & 0.13 & 0.05 & 0.11 & 0.04 & 0.59 & 0.12 \\
\hline 24 & 0.71 & 0.74 & 0.06 & 3.52 & 1.12 & 6.15 & 1.23 \\
\hline 25 & 2.13 & 1.54 & 1.81 & 0.39 & 2.35 & 8.22 & 1.64 \\
\hline 26 & 2.16 & 0.07 & 0.79 & 0.22 & 2.18 & 5.42 & 1.08 \\
\hline 27 & 0.23 & 0.96 & 1.63 & 1.24 & 3.32 & 7.38 & 1.48 \\
\hline 28 & 1.68 & 0.18 & 0.94 & 1.02 & 1.10 & 4.92 & 0.98 \\
\hline 29 & 2.04 & 1.63 & 0.21 & 1.03 & 0.03 & 4.94 & 0.99 \\
\hline 30 & 0.54 & 1.32 & 1.67 & 0.55 & 0.05 & 4.13 & 0.83 \\
\hline 31 & 1.46 & 1.56 & 0.95 & 0.57 & 0.48 & 5.02 & 1.00 \\
\hline 32 & 3.34 & 1.33 & 1.48 & 1.13 & 0.82 & 8.10 & 1.62 \\
\hline 33 & 1.64 & 1.75 & 1.04 & 0.37 & 0.78 & 5.58 & 1.12 \\
\hline 34 & 1.66 & 0.42 & 0.51 & 0.46 & 0.35 & 3.40 & 0.68 \\
\hline 35 & 2.31 & 2.19 & 1.92 & 1.75 & 0.13 & 8.30 & 1.66 \\
\hline 36 & 2.26 & 0.13 & 0.07 & 0.14 & 2.43 & 5.03 & 1.01 \\
\hline 37 & 2.29 & 2.19 & 0.05 & 0.13 & 0.18 & 4.84 & 0.97 \\
\hline 38 & 2.24 & 2.33 & 2.09 & 0.32 & 0.26 & 7.24 & 1.45 \\
\hline 39 & 0.99 & 1.81 & 0.19 & 0.58 & 0.90 & 4.47 & 0.89 \\
\hline 40 & 1.11 & 0.88 & 0.67 & 1.67 & 1.57 & 5.90 & 1.18 \\
\hline 41 & 1.84 & 0.44 & 0.36 & 0.11 & 0.16 & 2.91 & 0.58 \\
\hline 42 & 2.30 & 0.51 & 2.41 & 0.26 & 0.96 & 6.44 & 1.29 \\
\hline 43 & 0.41 & 1.21 & 0.99 & 1.81 & 0.71 & 5.13 & 1.03 \\
\hline 44 & 0.14 & 0.54 & 1.33 & 0.78 & 0.12 & 2.91 & 0.58 \\
\hline 45 & 1.83 & 1.59 & 1.64 & 1.16 & 0.19 & 6.41 & 1.28 \\
\hline 46 & 1.65 & 1.26 & 1.18 & 1.80 & 1.53 & 7.42 & 1.48 \\
\hline 47 & 1.93 & 1.10 & 1.29 & 1.91 & 1.55 & 7.78 & 1.56 \\
\hline 48 & 1.52 & 1.48 & 0.33 & 1.36 & 0.02 & 4.71 & 0.94 \\
\hline 49 & 1.43 & 1.36 & 1.28 & 1.18 & 0.89 & 6.14 & 1.23 \\
\hline 50 & 1.52 & 1.03 & 1.05 & 0.79 & 0.67 & 5.06 & 1.01 \\
\hline
\end{tabular}

Table 2. Measured film $O D, N O D$ and $M O D$.

In Table 2, the measured optical densities varied across the image of the mammograms during the measurements. This is expected, as the thickness and density of the tissues involved varies for each mammogram. The average of the measured
OD's is thus taken to be the mean optical density (MOD). Min OD 0.01, Max OD 3.64, Min NOD 0.58, Max NOD 10.36, Min MOD 0.12 and Max MOD 2.07. Table 3 presents the MOD and the estimated dose in accordance to the film 
serial numbers from 1 to 50 .

Table 3. MOD's and estimated dose.

\begin{tabular}{|c|c|c|c|c|c|}
\hline FILM S/N & MOD & Estimated dose (mGy) & FILM S/N & MOD & Estimated dose (mGy) \\
\hline 1 & 0.46 & 0.18 & 26 & 1.08 & 0.46 \\
\hline 2 & 1.37 & 0.62 & 27 & 1.48 & 0.68 \\
\hline 3 & 1.96 & 0.99 & 28 & 0.98 & 0.42 \\
\hline 4 & 2.07 & 1.07 & 29 & 0.99 & 0.42 \\
\hline 5 & 1.24 & 0.55 & 30 & 0.83 & 0.34 \\
\hline 6 & 0.12 & 0.04 & 31 & 1.00 & 0.43 \\
\hline 7 & 0.31 & 0.12 & 32 & 1.62 & 0.76 \\
\hline 8 & 0.21 & 0.08 & 33 & 1.12 & 0.48 \\
\hline 9 & 1.11 & 0.48 & 34 & 0.68 & 0.27 \\
\hline 10 & 1.64 & 0.78 & 35 & 1.66 & 0.79 \\
\hline 11 & 1.33 & 0.59 & 36 & 1.01 & 0.43 \\
\hline 12 & 0.35 & 0.13 & 37 & 0.97 & 0.41 \\
\hline 13 & 0.13 & 0.05 & 38 & 1.45 & 0.66 \\
\hline 14 & 0.66 & 0.27 & 39 & 0.89 & 0.37 \\
\hline 15 & 1.03 & 0.44 & 40 & 1.18 & 0.51 \\
\hline 16 & 1.12 & 0.48 & 41 & 0.58 & 0.23 \\
\hline 17 & 1.49 & 0.68 & 42 & 1.29 & 0.57 \\
\hline 18 & 1.39 & 0.63 & 43 & 1.03 & 0.44 \\
\hline 19 & 1.46 & 0.67 & 44 & 0.58 & 0.23 \\
\hline 20 & 1.77 & 0.86 & 45 & 1.28 & 0.57 \\
\hline 21 & 0.92 & 0.39 & 46 & 1.48 & 0.68 \\
\hline 22 & 0.80 & 0.33 & 47 & 1.56 & 0.72 \\
\hline 23 & 0.12 & 0.04 & 48 & 0.94 & 0.39 \\
\hline 24 & 1.23 & 0.54 & 49 & 1.23 & 0.54 \\
\hline 25 & 1.64 & 0.78 & 50 & 1.01 & 0.43 \\
\hline
\end{tabular}

In Table 3, the variations occur throughout the films in terms of the measured OD and the estimated absorbed dose. This was expected because the tissues and tissues densities were not the same for the patients undergoing the mammography screening and diagnosis. The Min MOD 0.12, Max MOD 2.07, Min estimated dose 0.04 and Max estimated dose 1.07 and the estimated absorbed dose are in agreement with those of the International Atomic Energy Agency (IAEA), guidance levels in X-ray guided medical interventional procedures [13].

Table 4 represents the descriptive statistics of the mean dose of the fifty (50) samples.

Table 4. Descriptive statistics of the mean dose of the fifty (50) samples and mean dose.

\begin{tabular}{lllllllll}
\hline $\begin{array}{l}\text { Mean dose of fifty (50) } \\
\text { samples (mGy) }\end{array}$ & \multicolumn{9}{l}{ Descriptive statistics of the fifty (50) sample doses (mGy) } \\
\cline { 2 - 8 } & Min & Max & Range & SD & Kurtosis & $\mathbf{1}^{\text {st }} \mathbf{Q}$ & $\mathbf{3}^{\text {rd }} \mathbf{Q}$ \\
\hline 0.48 & 0.04 & 1.07 & 1.03 & 0.24 & 2.77 & 0.34 & 0.66 \\
\hline
\end{tabular}

In Table 4, Mean dose 0.48, Min dose 0.04, Max dose 1.07, Range 1.03 , SD 0.24 , Kurtosis $2.77,1^{\text {st }} \mathrm{Q} 0.34$ and $3^{\text {rd }}$ Q 0.66.. The Min and Max dose of the fifty (50) samples depicts a good trend in the consistency of the amount of radiation exposure across the mammograms, as required by the dose reference level for mammography. The Range was also close to the Max dose, which gives an account of the closeness of values across the estimated dose. The SD is found to be less enough, which shows that the estimated dose values are not too diverge from the Mean dose as a reference value and this gives account for good measurement throughout the work. The Kurtosis value shows a normal distribution of the accuracy of the OD measurements and the estimated doses across the mammogram samples, that is, the normal distribution is a symmetrical distribution with wellbehaved tails. The $1^{\text {st }} \mathrm{Q}$, is the median of the lower half of the data set. This means that about $25 \%$ of the dose lie below $\mathrm{Q}_{1}$ and about $75 \%$ lie above $\mathrm{Q}_{1}$. The $3^{\text {rd }} \mathrm{Q}$, is the median of the upper half of the data set. This means that about $75 \%$ of the dose lie below $\mathrm{Q}_{3}$ and about $25 \%$ lie above $\mathrm{Q}_{3}$. These results in Table 4 were in good agreement with the Nigeria Basic Ionizing Radiation Regulation (NBIRR) [14] and the International Commission on Radiological Protection (ICRP) [15].

The figures represent the plot and the results of MOD's against $X$, the estimated doses, from curve fitting using the MATLAB. 


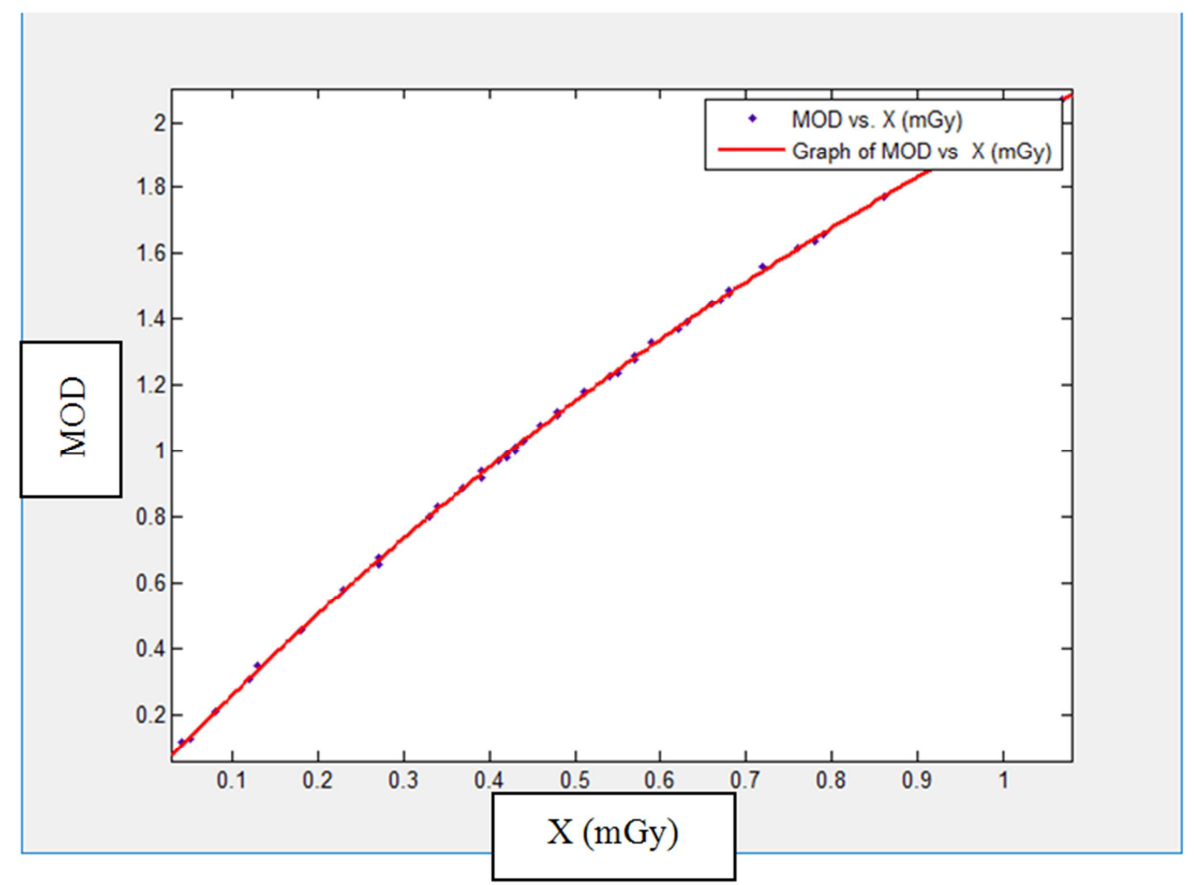

Figure 1. Graph of MOD vs $X(m G y)$.

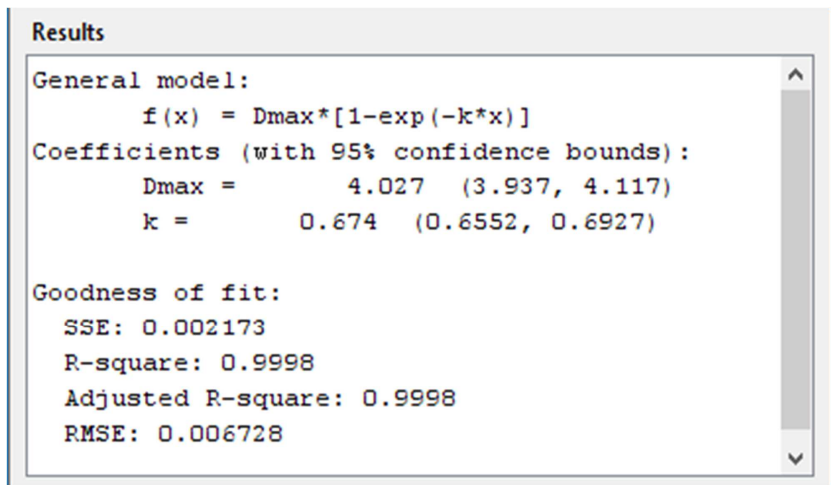

Figure 2. Results of the curve fitting.

Figure MOD vs X (mGy) show a good exponential trend in the measured OD and the estimated dose. The higher the OD value, the greater the amount of estimated dose. Results of the fit, contains the General model $\mathrm{f}(\mathrm{x})$, used in estimating the doses, Coefficients (with 95\% confidence bounds) as $\mathrm{D}_{\max }$ fixed at 4.027 , interval of between 3.937 to 4.117 and $\mathrm{k}$ fixed at 0.674 , interval of between 0.6552 to 0.6927 . The Goodness of fit has; Sum of Squares Due to Error (SSE) 0.002173 , which is close to 0 , indicating that the model has a smaller random error component, and the fit will be more useful for prediction. $\mathrm{R}$ - square 0.9998 , a value closer to 1 , indicating that a greater proportion of variance is accounted for by the model. Adjusted R - square 0.9998, this is closer to 1 , indicating a better fit. Root Mean Square Error, 0.006728 , this is close to 0 , which indicates a fit that is more useful for prediction.

\section{Conclusion}

In Table 3, the MOD can be seen to vary from one film to the other. The higher the MOD, the greater the value of the estimated dose. This depicts a good trend in the dosage application to patients undergoing screening and diagnosis. It is however important that ALARA (as low as reasonably achievable) principle of X-ray radiation dose be used for the purpose of mammography screening and diagnosis. Even those who believe that imaging radiation has a long-term risk of cancer, concede that this risk is extremely small. It is suggested that leading radiology, health physics and other medical societies, publish position statements to educate physicians and patients regarding the possibility risks of cancer from medical imaging radiation. The QA and QC approach to screening and diagnosis dosage usage in mammography can thus be derived from this work, to safeguard against excess dose or injury to patients and personnel.

\section{Acknowledgements}

The authors will like to thank the University of Benin Teaching Hospital (UBTH), Benin City, Edo State, Nigeria and the staffs of the Radiology department of the hospital for their efforts in providing the mammography films for use, with other advice and suggestions.

\section{References}

[1] Akinlade, B. I., Odefemi, F. B. and Farai, I. P. (2016). Overview of radiation dose to patients from medical $\mathrm{X}$ - ray examinations in Nigeria. Afr. J. Med. Sci. 45, 23 - 29.

[2] NCRP (National Council on Radiation Protection and Measurement) (1987). Ionizing radiation exposure of the population of the United States. NCRP 1987, report 93. 
[3] Ajayi, I. R. and Akinwumi, A. (2000). Measurement of entrance skin doses to patients in four common diagnostic examinations by thermo luminescence dosimetry in Nigeria. Radiat. Prot. Dosim. 2000; 87(3): 213 - 220.

[4] Boerma M, Nelson GA, Sridharan V, Mao XW, Koturbash I, Hauer-Jensen M. Space radiation and car-diovascular disease risk. World J Cardiol. (2015). 7(12):882-8. Epub 2016/01/06. https://doi.org/10.4330/wjc.v7.i12.882 PMID: 26730293.

[5] Lu T, Zhang Y, Wong M, Feiveson A, Gaza R, Stoffle N, et al. Detection of DNA damage by space radiation in human fibroblasts flown on the International Space Station. Life Sciences in Space Research. (2017). 12 (Supplement C):24 31. https://doi.org/10.1016/j.1ssr.2016.12.004.

[6] Dobrzynski L, Fornalski KW, Feinendegen LE. Cancer Mortality Among People Living in Areas With Various Levels of Natural Background Radiation. Dose Response. (2015). 13(3):1559325815592391. Epub 2015/12/18. https://doi.org/10.1177/1559325815592391 PMID: 26674931.

[7] Medlin D, Heffron W, Siegel A, Wilson K, Klingenberger A, Gall A, et al. Development of an X-ray irradiation port for biomedical applications at the CUEBIT facility. Journal of Physics: Conference Series. (2015). 583(1):012048.

[8] Mykyta Sokolov and Ronald Neumann (2016). Global Gene Expression Alterations as a Crucial Constituent of Human Cell
Response to Low Doses of Ionizing Radiation Exposure. Int. J. Mol. Sci. (2016). 17, 55.

[9] Mervyn D Cohen (2018). Is there a risk of getting cancer from radiation from medical diagnostic imaging? J Radiol Med Imaging. 2018; 1: 1005.

[10] GAMMEX (2016). Film Densitometer Peripheral Technical Manual, Model 5336. GAMMEX Middleton, WI, USA.

[11] Scarlat, F., Scarisoreanu, A., Oane, M., Mitre, E., Badita, E. (2008). Determination of Absorbed Dose Using a Dosimetric Film. IX Radiation Physics \& Protection Conference, 15 - 19 November, 2008, Nasr City, Egypt. 313 - 321.

[12] Artur, T. (2003). Determination of Absorbed X-ray Radiation Dose in X-ray Diagnostics and Imaging. J. Med. Sci. 1 (2); 31-36.

[13] IAEA (International Atomic Energy Agency) (Safety Reports Series No. 59). Establishing Guidance Levels in $X$ - ray Guided Medical Interventional Procedures: A Pilot Study. Safety Reports Series No. 59.

[14] NBIRR (Nigeria Basic Ionizing Radiation Regulation) (2003).

[15] ICRP (International Commission on Radiological Protection) (1996). Radiological Protection and safety in Medicine, Publication 73 (Oxford and New York: Pergamon Press). 